\title{
GRB Repetition Limits from Current BATSE Observations
}

\author{
Jon Hakkila*, Charles A. Meegan ${ }^{\ddagger}$, Geoffrey N. Pendleton ${ }^{\dagger}$, \\ Michael S. Briggs ${ }^{\dagger}$, John M. Horack ${ }^{\ddagger}$, Dieter H. Hartmann ${ }^{\circ}$, and \\ Valerie Connaughton ${ }^{\star}$ \\ * Mankato State University, Mankato MN 56002-8400 \\ ${ }^{\ddagger}$ NASA/Marshall Space Flight Center, Huntsville, AL 35824 \\ ${ }^{\dagger}$ University of Alabama in Huntsville, Huntsville, AL 35899 \\ ${ }^{\circ}$ Clemson University, Clemson, SC 29634 \\ ${ }^{\star}$ National Research Council at NASA/MSFC, Huntsville, AL 35824
}

\begin{abstract}
Revised upper limits on gamma-ray burst repetition rates are found using the BATSE 3B and 4B catalogs. A statistical repetition model is assumed in which sources burst at a mean rate but in which BATSE observes bursts randomly from each source.
\end{abstract}

\section{INTRODUCTION}

Regardless of the gamma-ray burst distance scale, the question of whether bursters repeat (e.g. [8] [11]) is of great importance to our understanding of them: their energy production mechanisms are constrained by repetition. There is strong statistical evidence to indicate that bursters are not observed to repeat on timescales of days to months ( [9] [4] [2]), but the recent identification of four gamma-ray bursts close together in both location and time [3] has left this possibility open for at least some bursters. Of equal interest is the possibility that they might repeat on timescales of years or longer, and that repetition might take some form where they do not burst steadily.

Detection of repetition is complicated by burst localization errors (do two bursts belong to the same source?) and by incomplete angular sky exposure and limited trigger efficiency (what fraction of source repetitions is observed?). The repetition question can be studied using two observational parameters [6] [4]; $f$ and $\langle\nu\rangle$. The variable $f$ represents the fraction of sources that produce more than one detected burst, while $\langle\nu\rangle$ represents the mean number of detected bursts per source producing more than one detected burst. 


\section{ANALYSIS}

Localization Errors. Localization errors are modeled as a combination in quadrature of a $1.6^{\circ}$ systematic error and a statistical error. The statistical error is a combination of a fluence-dependent mean and a fluence-dependent random component obtained from the BATSE 4B Catalog.

Sky Exposure. The sky exposure used is that listed in the BATSE 4B Catalog. It should be noted that this exposure is only valid for non-overwriting bursts, so that overwriting bursts must be removed from the dataset.

Trigger Efficiency. The effects of trigger efficiency on repetition are not included in this analysis. This is due in part to calibration work still in progress (see Pendleton, Hakkila, \& Meegan, this conference) and in part to the modeldependent way in which the repetition luminosity function enters into the analysis (e.g. [1] [4]).

The Burst Catalogs. For this analysis we choose to independently analyze the BATSE 3B [7] and 4B catalogs. The catalogs have had all overwriting bursts removed, leaving 1060 3B bursts and 1554 4B bursts. We recognize that $4 \mathrm{~B}$ bursts have been detected using several triggering energy channel combinations; this complicates the analysis in complex ways not modeled here. The $3 \mathrm{~B}$ analysis does not suffer from this effect.

Clustering Statistic. The value of the Two-Point Angular Correlation Function found within $7.2^{\circ}\left[\operatorname{TPACF}\left(\theta \leq 7.2^{\circ}\right)\right]$ is used as the clustering test for this analysis. This angle has been found to contain an optimum signal for repeating bursts [10] due to the overall distribution of burst localization probabilities.

Repetition Models. Two models are used; a Euclidean model and a model based on an Einstein-de Sitter cosmology with the faintest bursts at redshift $z=2$ (Hereafter referred to as the ES2 model). Both spatial models have only one free parameter: it is assumed that bursters burst with a mean intrinsic repetition rate $R_{\text {int }}$ (repetitions source ${ }^{-1}$ year $^{-1}$ ), but that equal probabilities exist that any burster might be the next one to burst. By varying $R_{\text {int }}$, various fractions of the bursts $f$ are found to belong to clusters composed of various numbers of detected bursts $\nu$ (having an average value $\langle\nu\rangle$ ), which corresponds to a mean observed repetition rate $R_{\text {obs }}$. In the limit of very low mean intrinsic repetition rates $\left(R_{\text {int }} \approx 0\right)$, these models are essentially the same as non-repeating models, since no clusters of two or more bursts will be produced during the BATSE operating time. In Euclidean space, $R_{\text {int }}$ is always less than $R_{\text {obs }}$, since the overall exposure is less than unity. In the case of the ES2 model, $R_{\text {int }}$ is assumed to be constant in the comoving frame. More distant ES2 bursters appear to repeat at a slower rate due to time dilation, so that a higher $R_{\text {int }}$ is needed to produce a Euclidean equivalent $R_{\text {obs }}$.

Monte Carlo Simulations. Monte Carlo simulations are performed while varying the free parameter $R_{\text {int }}$. It is found from the Monte Carlo simulations that

$$
R_{\text {obs }}=0.481 R_{\text {int;Euclidean }}=0.310 R_{\text {int } ; \mathrm{ES} 2}
$$


Thus, there is no real difference between the Euclidean and ES2 cosmological models in terms of the repetition observables.

The Monte Carlo simulations allow $\langle\nu\rangle$ vs $f$ plots to be produced for both the BATSE 3B and 4B catalogs. These are presented in Figure 1, along with the corresponding mean observed repetition rates. $R_{\text {obs }}$ from $\langle\nu\rangle$ and $f$ differs between the two catalogs because the exposure differs. The $4 \mathrm{~B}$ exposure is slightly better than the $3 \mathrm{~B}$ exposure; if the two were identical the rates would coincide on the plots. If the rates were identical, then the longer $4 \mathrm{~B}$ Catalog would produce more cluster composed of larger numbers of bursts. This effect is enhanced by the better $4 \mathrm{~B}$ exposure, so that even more and larger clusters should be produced. It is therefore expected that repetition rates of the type described should be easier to identify in the 4B Catalog than in the 3B Catalog.

Repetition Limit Procedure. The BATSE 3B and $4 \mathrm{~B}$ values of the TPACF within $7.2^{\circ}$ of one another $\left[\operatorname{TPACF}\left(\theta \leq 7.2^{\circ}\right)\right]$ are slightly negative (the $3 \mathrm{~B}$ value of -0.070 is $1.5 \sigma$ from the isotropic value of zero, while the $4 \mathrm{~B}$ Catalog value of -0.035 is $1.1 \sigma$ from isotropy), indicating a depletion of close burst pairs. Since models with nonzero repetition rates produce positive values of this statistic, both BATSE catalogs are most consistent with no repetition.

We can also ask how consistent the results are with different mean observed repetition rates $R_{\text {obs }}$, so that we can get some idea concerning upper limits allowed on repetition rates. To do this, the $\operatorname{TPACF}\left(\theta \leq 7.2^{\circ}\right)$ measurement (with associated error distribution; assumed Gaussian) at each $R_{\text {obs }}$ can be compared to the minimum expected value of $\operatorname{TPACF}\left(\theta \leq 7.2^{\circ}\right)=0$ at $R_{\text {obs }}=0$ (assuming that TPACF $=0$ is the correct value). This sensitivity calculation can provide some idea as to the maximum rate $R_{\text {obs }}$ that would go undetected by the BATSE instrument.

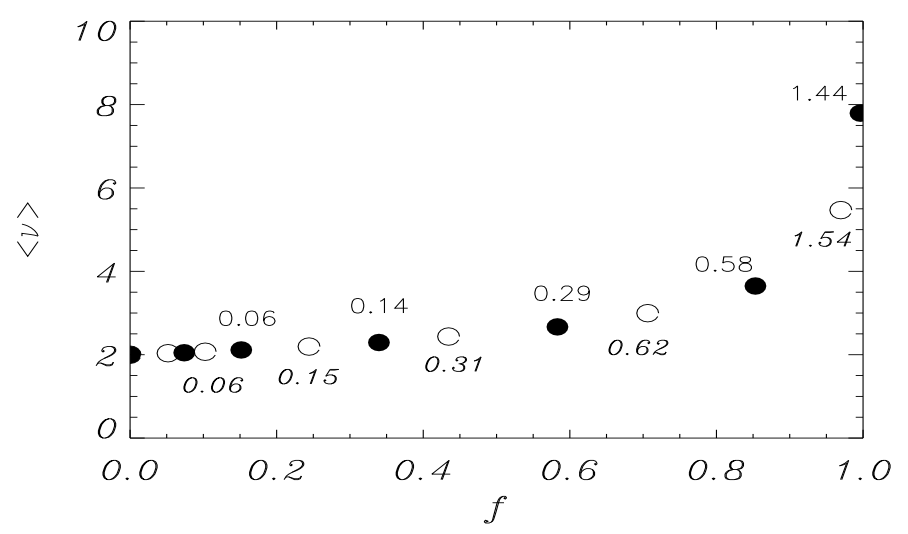

FIGURE 1. Correspondence between the mean observed repetition rate $R_{\text {obs }}$ and the Meegan $f$ and $\langle\nu\rangle$ parameters for the repetition modelin question. Specific rates $R_{\text {obs }}$ (bursts source ${ }^{-1}$ year $^{-1}$ ) are indicated for both the BATSE 3B (open circles; italic lettering) and 4B (closed circles; roman lettering) catalogs. The shape of the $\langle\nu\rangle$ vs. $f$ relationships seen here is characteristic of this repetition model. 
TABLE 1. Limits on mean observed and intrinsic repetition rates (in units of bursts source ${ }^{-1}$ year $^{-1}$ ) for Euclidean and ES2 models. Limits indicate rates for which the probability is $90 \%$ that $\operatorname{TPACF}\left(\theta \leq 7.2^{\circ}\right) \geq 0$, as opposed to the negative $\operatorname{TPACF}\left(\theta \leq 7.2^{\circ}\right)$ values obtained from the BATSE $3 \mathrm{~B}$ and $4 \mathrm{~B}$ catalogs.

\begin{tabular}{||c|c|c|c||}
\hline Catalog & $R_{\text {obs }}$ Limit & $R_{\text {int;Euclidean Limit }}$ & $R_{\text {int;RS2 Limit }}$ \\
\hline BATSE 3B & 0.15 & 0.31 & 0.48 \\
BATSE 4B & 0.05 & 0.10 & 0.16 \\
\hline
\end{tabular}

\section{CONCLUSIONS}

Figure 2 indicates the probability that $\operatorname{TPACF}\left(\theta \leq 7.2^{\circ}\right) \geq 0$ assuming different $R_{\text {obs }}$ rates in the BATSE $3 \mathrm{~B}$ and BATSE $4 \mathrm{~B}$ catalogs. At the $90 \%$ confidence level, $R_{\text {obs }} \leq 0.15$ bursts source $^{-1}$ year $^{-1}$. The larger data set of the BATSE 4B Catalog would place even greater constraints on the observed repetition rate, if one considers the different triggering criteria used in this catalog to be unimportant. For the $4 \mathrm{~B}$ Catalog, it is unlikely that $R_{\text {obs }} \geq 0.04$ bursts source $^{-1}$ year $^{-1}$. This indicates that repetition is either absent or that sources only repeat rarely on the timescales of the BATSE catalogs. These $90 \%$ probabilities correspond to model-dependent limits on the mean intrinsic repetition rates, which are indicated in Table 1.

In a previous work, we [4] quoted similar types of limits on the Euclidean mean intrinsic repetition rate, finding that $R_{\text {obs }} \geq 0.05$ bursts source $^{-1}$ year $^{-1}$ was unlikely in the BATSE 3B Catalog. Our revised 3B 90\% upper limit is higher at $R_{\text {obs }}=0.31$ bursts source ${ }^{-1}$ year $^{-1}$, in part due to a more direct definition of our

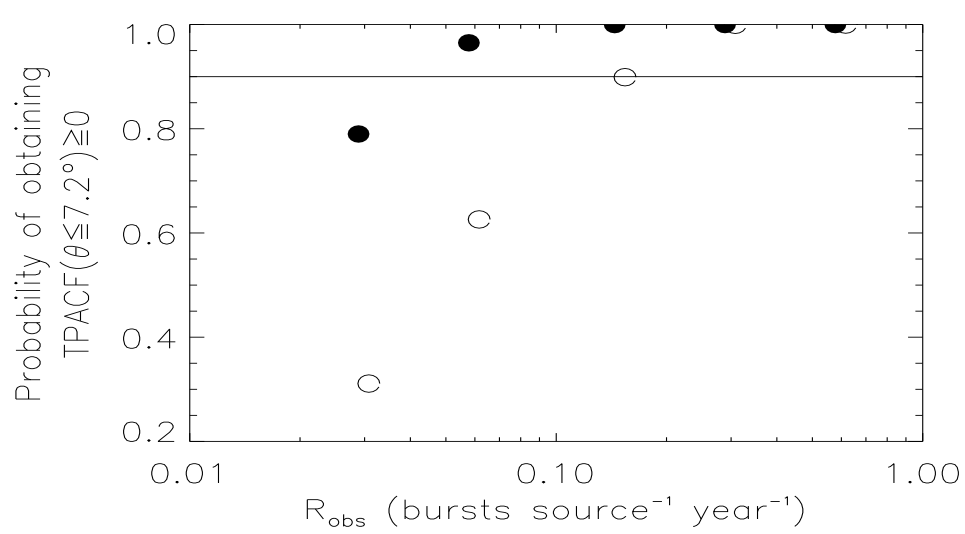

FIGURE 2. Probability of obtaining a value of $\operatorname{TPACF}\left(\theta \leq 7.2^{\circ}\right) \geq 0$ vs. mean observed repetition rate $R_{\text {obs }}$. The plot indicates that the larger $4 \mathrm{~B}$ data set (closed circles) is more sensitive to detecting repetition of the type described, than the $3 \mathrm{~B}$ data set (open circles). Unknown effects in the $4 \mathrm{~B}$, such as that of triggering on different energy channels, have not been taken into account in this analysis. 
statistical significance, and in part indicating that BATSE is not as sensitive to detecting repetition as a result of the improved sky exposure analysis. The new sky exposure requires that overwrites be excluded; with fewer bursts, higher repetition rates are needed to be detected above the random background. The new exposure is also higher than that estimated before, indicating that more of any intrinsic repetitions should be detected. By selecting fewer bursts from sources, cluster sizes tend to be smaller. Thus $\langle\nu\rangle$ is smaller for a given repetition rate, and is more difficult to detect.

Our analysis technique could still be more sensitive to detecting repetition than a recent technique that combines localization probabilities from multiple experiments to obtain smaller localization regions [5]. That technique limits its database to bursts detected by both experiments, so that the sky exposure and trigger efficiency for the combined experiment must then be found by multiplying these detection probabilities together. We have shown here that limits on repetition rates depend both on localization uncertainty and sky exposure.

Our repetition model allows that some observed clusters can be large, and in fact suggests that is it possible for the October 1996 burst "cluster" [3] to have come from one source. From the upper limit on the repetition rate described above, it is expected that at most 29 clusters of 3 bursts and 4 clusters of 4 bursts should be observed in the BATSE 3B Catalog. The tighter limits on 4B Catalog repetition, however, indicate that only as many as 19 clusters of 3 bursts and 1 cluster of 4 bursts are expected.

\section{REFERENCES}

1. Band, D. J., 1994, ApJ, 422, L75.

2. Brainerd, J. J., 1996, ApJ, 473, 974.

3. Connaughton, V. et al., 1997, in Proc. 18th Texas Symposium, ed. A. Olinto, in press.

4. Hakkila, J., et al., 1996, in Gamma-Ray Bursts, ed. C. Kouveliotou, M. S. Briggs, \& G. J. Fishman, (AIP: New York), p. 392.

5. Kippen, R. M. et al., 1998, (this conference).

6. Meegan, C. A. et al., 1995, ApJ, 446, L15.

7. Meegan, C. A. et al., 1996, ApJS, 106, 65.

8. Quashnock, J. M., \& Lamb, D. Q., 1993, MNRAS, 265, L45.

9. Tegmark, M. et al., 1996, ApJ, 466, 757.

10. Vo, V., 1994, Master's Thesis, Mankato State University, Mankato, MN.

11. Wang, V. \& Lingenfelter, R. E., 1995, ApJ, 441, 747. 